\title{
ROTATION PROFILE OF A STRING-SEEDED HALO.
}

\author{
W. H. Zurek ${ }^{1}$ and P. J. Quinn ${ }^{2}$ \\ 1. Theoretical Astrophysics \\ LANL, Los Alamos, NM 87545. \\ 2. Space Telescope Science Inst. \\ Baltimore, MD 21218.
}

We have used an $\mathrm{N}$-body code to simulate the collapse of a wake left by a loop of cosmic string in a cold dark matter universe $(\Omega=$ $1, h=0.5$ ). Initial perturbations were introduced via the Zeldovich approximation in a high-resolution sphere of radius $1.5 \mathrm{Mpc}$ (comoving) filled with a lattice of $\sim 10^{4}$ points masses of $1.38 \times 10^{8} M_{\odot}$. The top plot shows, at $z=24$, a wake of a loop of $M_{S}=3 \times$ $10^{8} M_{\odot}$ which has moved up and left through the center of the sphere with $z_{\text {eq }}$ velocity $v=$ $0.01 c$ directed at an $18^{\circ}$ angle out of the plane of the figure. The collapsed object (center) is quite prolate. The outer shell of more massive particles is now also visible. The spherically averaged rotation profile of the "halo" is shown in the bottom diagram. The almost flat portion of the rotation curve corresponds to the size of the wake. There is also evidence for a soft core of $\sim 10 \mathrm{kpc}$ in radius (our dynamical softening was $3 \mathrm{kpc}$.). While the adopted initial velocity and mass are appropriate for loops with galaxy - galaxy separations, a one loop - one object correspondence is not a good approximation on the galactic scale. Moreover, baryons settling in the core of the halo would have steepened the profile and made it incompatible with observations. Halos seeded by several loops may have rotation profiles more compatible with those observed. The results of our simulations may be applied - after an appropriate rescaling - to Abell clusters. There one loop typically seeds a single cluster. String-seeded Abell clusters should be therefore significantly prolate.
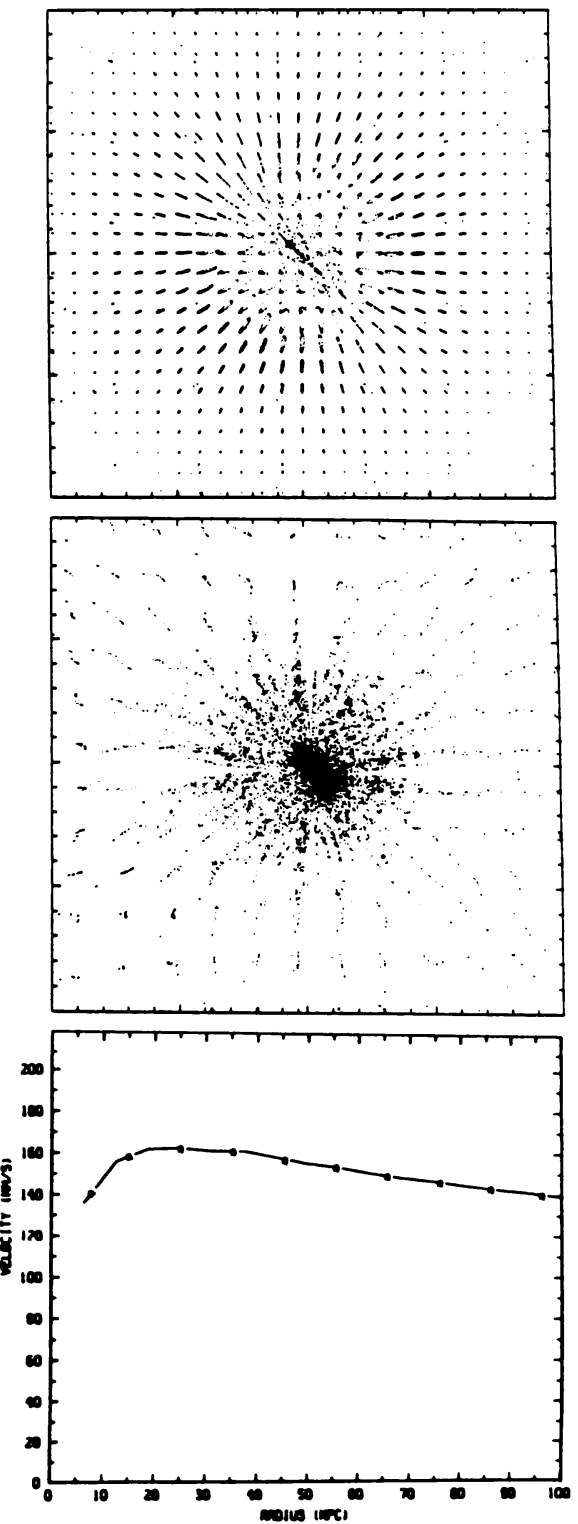